
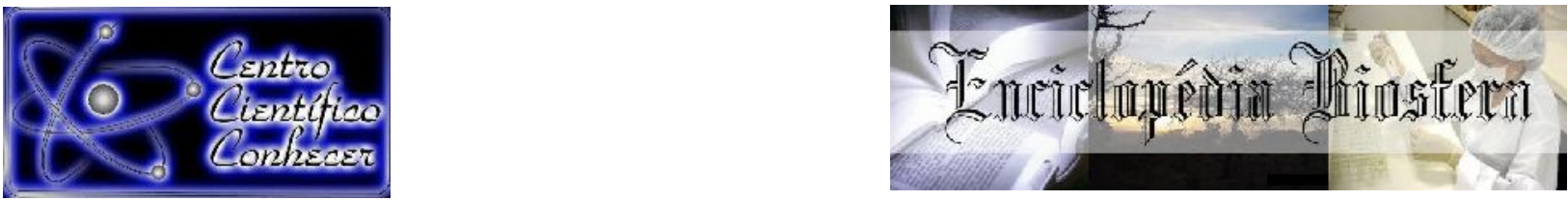

\title{
PERFIL DOS USUÁRIOS DE SUBSTÂNCIAS PSICOATIVAS ATENDIDOS EM UM CENTRO DE ATENÇÃO PSICOSSOCIAL DO ACRE
}

Jhonnatan Antonio Gurgel dos Santos ${ }^{1}$, Fábio Sena de Lima ${ }^{1}$, Thiago dos Santos da Silva ${ }^{1}$, Rafael Tavares Lima Izel ${ }^{1}$, Eder Ferreira de Arruda $^{2}$

1. Bacharéis em Enfermagem pelo Centro Universitário UNINORTE, Rio Branco-AC, Brasil.

2. Docente do Centro Universitário UNINORTE, Rio Branco-AC, Brasil. E-mail: ederarrud@gmail.com.

Recebido em: 04/10/2019 - Aprovado em: 30/11/2019 - Publicado em: 15/12/2019 DOI: 10.18677/EnciBio_2019B45

\begin{abstract}
RESUMO
Atualmente, vivencia-se um crescimento acelerado do uso de substâncias psicoativas repercutindo em todos os segmentos sociais, por isso este estudo teve como objetivo descrever o perfil de um grupo de usuários de substâncias psicoativas atendidos em um centro de referência do município de Rio Branco-Acre. Realizou-se um estudo descritivo do tipo transversal, com 31 indivíduos, em maio de 2019, atendidos em um centro de referência nesse município, no qual foram coletados dados sobre as características sociodemográficas e os fatores relacionados da dependência química. Observou-se que a maioria dos indivíduos era do sexo masculino $(87,1 \%)$, na faixa etária de 36 a 45 anos de idade (32.3\%), tinha cursado apenas o ensino fundamental $(45,2 \%)$ e possuía antecedentes familiares de uso de substâncias psicoativas $(64,5 \%)$. O álcool foi identificado como a primeira droga utilizada pela maioria dos indivíduos $(41,9 \%)$, sendo que a maior parte usou drogas pela primeira vez por curiosidade $(48,4 \%)$, costumava usar duas ou mais drogas ao mesmo tempo $(77,4 \%)$, se sentiam excluídos da sociedade por ser dependente químico $(77,4 \%)$, avaliaram sua condição de saúde como regular $(58,1 \%)$, buscaram o tratamento por iniciativa própria $(74,2 \%)$, recebiam apoio familiar $(51,6 \%)$ e definiram a assistência prestada pelo centro psicossocial como muito boa $(45,2 \%)$. Desse modo, conhecer o perfil dos usuários pode auxiliar e orientar as equipes de saúde na promoção de ações e serviços preventivos e assistenciais de acordo com a realidade dos pacientes.
\end{abstract}

PALAVRAS-CHAVE: abuso de substâncias psicoativas; perfil de saúde; sistemas de apoio psicossocial.

\section{USER PROFILE OF PSYCHOACTIVE SUBSTANCES AT A PSYCHOSOCIAL ATTENTION CENTER IN RIO BRANCO MUNICIPALITY, ACRE}

\begin{abstract}
Currently, there is an accelerated growth in the use of psychoactive substances affecting all social segments, so this study aimed to describe the profile of a group of users of psychoactive substances seen at a referral center of the city of Rio Branco-
\end{abstract}


Acre. A cross-sectional descriptive study was carried out with 31 individuals, in May 2019, attended at a referral center in this municipality, where data on sociodemographic characteristics and related factors of chemical dependence were collected. Most of the individuals were male $(87.1 \%)$, aged 36 to 45 years $(32.3 \%)$, had only attended elementary school (45.2\%) and had a family history. use of psychoactive substances (64.5\%). Alcohol was identified as the first drug used by most individuals (41.9\%), most of them using drugs for the first time out of curiosity $(48.4 \%)$, used to use two or more drugs at the same time $(77,4 \%)$, felt excluded from society because they were chemically dependent $(77.4 \%)$, rated their health condition as fair $(58.1 \%)$, sought treatment on their own $(74.2 \%)$, received support family (51.6\%); and defined the care provided by the psychosocial center as very good $(45.2 \%)$. Thus, knowing the profile of users can help and guide health teams in promoting preventive and care actions and services according to the reality of patients.

KEYWORDS: abuse of psychoactive substances; health profile; psychosocial support systems.

\section{INTRODUÇÃO}

O consumo de substâncias psicoativas faz parte da própria história da humanidade estando presente em diferentes culturas, apresentando distintas funcionalidades, cenários e padrões de uso. Contudo, o intenso abuso de drogas a partir do século XX se configurou como um importante problema de saúde pública mundial (WHITEFORD et al., 2013).

Segundo dados do relatório mundial sobre drogas de 2019, cerca de 35 milhões de pessoas em todo o mundo sofrem de transtornos por uso de drogas, enquanto apenas uma em cada sete pessoas recebe tratamento (UNODC, 2019).

No Brasil, resultados do III Levantamento Nacional sobre o Uso de Drogas pela População Brasileira (III LNUD) revelaram que 3,2\% dos brasileiros já usaram substâncias ilícitas pelo menos uma vez na vida, sendo que esse percentual é muito maior entre os homens $(5,0 \%)$ e entre os jovens entre 18 e 24 anos $(7,4 \%)$. As substâncias ilícitas mais consumidas pelos brasileiros foram a maconha $(7,7 \%)$ e a cocaína (3,1\%) (BASTOS et al., 2017).

O abuso de drogas lícitas e ilícitas é uma preocupação por ocasionar graves prejuízos sociais, psíquicos e biológicos e os motivos que levam ao aumento do uso dessas substâncias são diversos e complexos (ELICKER et al., 2015).

As consequências do uso de drogas vão além dos danos individuais e orgânicos, uma vez que interferem diretamente no contexto familiar, transformando os membros da família em codependentes, causando desagregação familiar e sofrimento psíquico e emocional (ALVARES et al., 2014).

Neste contexto, é importante conhecer as características dos usuários e os fatores relacionados à sua dependência química no intuito de estabelecer medidas de atenção à saúde destes indivíduos. Dessa forma, o presente estudo teve como objetivo descrever o perfil de um grupo de usuários de substâncias psicoativas atendidos em um centro de referência do município de Rio Branco-Acre.

\section{MATERIAIS E MÉTODOS}

Tratou-se de um estudo observacional do tipo transversal com abordagem quantitativa realizado com dependentes químicos atendidos no Centro de Atenção Psicossocial e outras Drogas (CAPS-AD III) do município de Rio Branco, Acre. 
Segundo o último censo realizado pelo Instituto Brasileiro de Geografia Estatística (IBGE), o município de Rio Branco está localizado no extremo oeste da Amazônia, a área territorial tem $8.834,942 \mathrm{~km}^{2}$ e possui população estimada de 407.319 pessoas em 2019 (IBGE, 2019).

A amostragem foi não probabilística, por conveniência, sendo selecionados aleatoriamente 31 indivíduos atendidos no CAPS-AD III durante o mês de maio de 2019. Foram incluídos os indivíduos de ambos os sexos, com idade igual ou superior a 18 anos e que voluntariamente aceitaram participar da pesquisa assinando 0 Termo de Consentimento Livre Esclarecido (TCLE). Foram excluído os indivíduos que não tinham condições de responder e/ou compreender o questionário no momento da entrevista.

Para a coleta de dados, foi aplicado um questionário sobre as características sociodemográficas e os fatores relacionados à dependência química. O programa estatístico Statistical Package for the Social Sciences (SPSS) versão 21.0 foi utilizado para a digitação, revisão e análise dos dados onde foram calculadas as frequências absolutas e relativas para as variáveis categóricas.

A presente pesquisa foi aprovada pelos Comitês de Ética em Pesquisa da União Educacional do Norte - UNINORTE, com o parecer № 3.260 .940 e CAAE № 08638919.1.0000.8028.

\section{RESULTADOS E DISCUSSÃO}

Dos 31 indivíduos atendidos no CAPS-AD III, a maioria era do sexo masculino (87,1\%), (Tabela 1). Igualmente, no trabalho de Cantarelli et al. (2014), que analisaram o perfil de usuários de substancias psicoativas em um hospital universitário do Rio Grande do Sul, entre outubro de 2009 a outubro de 2010, observaram que os homens eram os principais usuários de substâncias psicoativas $(94,39 \%)$. De forma similar, na pesquisa de Almeida et al. (2014), realizada em João Pessoa - PB, no ano de 2013, também verificaram que a maior proporção de usuários dessas substâncias, eram do sexo masculino $(86,68 \%)$. Segundo Oliveira et al. (2007), os homens estão predispostos a usar drogas mais cedo durante a vida e, em maior abundância e maior período de tempo quando comparados ao sexo oposto.

TABELA 1 - Características sociodemográficas dos usuários de substâncias psicoativas atendidos em um centro de referência de Rio Branco, Acre, Brasil, 2019.

\begin{tabular}{lcc}
\hline Variável & $\mathbf{N}$ & $\%$ \\
\hline Sexo & & \\
Masculino & 27 & 87,1 \\
Feminino & 04 & 12,9 \\
Faixa etária (anos) & & \\
$<25$ & 05 & 16,1 \\
$25-35$ & 06 & 19,4 \\
$36-45$ & 10 & 32,3 \\
$46-60$ & 09 & 29,0 \\
$>60$ & 01 & 3,2 \\
Escolaridade & & \\
Não alfabetizado & 04 & 12,9 \\
Ensino fundamental & 14 & 45,2 \\
Ensino médio & 11 & 35,5 \\
Ensino superior & 02 & 6,5 \\
\hline Total & $\mathbf{3 1}$ & $\mathbf{1 0 0 , 0}$ \\
\hline
\end{tabular}


Em relação à idade, a maioria estava na faixa etária de 36 a 45 anos de idade (32.3\%), (Tabela 1). De forma semelhante, no estudo de Mastroianni et al. (2016), realizada no CAPS AD de uma cidade no interior de São Paulo, encontraram que a maioria dos indivíduos estavam com idade igual ou superior a 35 anos $(41,9 \%)$. Diferentemente, na pesquisa realizada por Morais (2018), na cidade de São Luís-MA em 2018, foi identificado que a maior parte dos usuários se encontrava na faixa etária entre 25 e 29 anos (59,9\%).

De acordo com Capistrano et al. (2013), as diferentes formas terapêuticas empregadas no tratamento de dependentes químicos, têm melhor efeito quando os pacientes apresentam idade mais avançada e fazem uso de drogas há muito tempo, tendo em vista que em consequência do uso de drogas ao longo da vida já vivenciaram grande sofrimento.

No que diz respeito à escolaridade, a maioria dos usuários tinham cursado apenas o ensino fundamental (45,2\%), (Tabela 1). Em estudo similar Almeida e Luis (2017), em uma cidade do interior, na região Centro-Oeste do estado de Minas Gerais, também constataram que a maioria dos dependentes apresentava apenas o ensino fundamental (47\%). De igual modo, em uma pesquisa desenvolvida por Almeida et al. (2014), identificaram que a maior parte dos usuários possuía somente o nível fundamental $(56,8 \%)$.

Para Pechansky et al. (2004), o uso de drogas acarreta uma série de danos cognitivos como dificuldade na memorização e formulação de pensamentos, portanto dificultando o aprendizado e originando o abandono escolar.

Conforme a tabela 2, a maior parte dos atendidos tinham antecedentes familiares com uso de substâncias psicoativas (64,5\%), (Tabela 2). Validando esta informação Marcon et al. (2015), na cidade de Cuiabá-MT, no ano de 2012, identificaram que $70,3 \%$ dos usuários de substâncias psicoativas apresentavam antecedentes de usuários no núcleo familiar, sendo mais frequentes pais e irmãos. Resultado diferente foi encontrado na pesquisa desenvolvida por Almeida et al. (2014), na qual a maioria $(61,33 \%)$ informaram não ter nenhum familiar usuário de drogas.

Para Marcon et al. (2015), estão mais sujeitas ao uso de drogas pessoas que estão submetidas a conjunções familiares com déficit de diálogo, relacionamentos insatisfatórios, incompreensão e abuso de substâncias psicoativas.

No que se diz respeito ao contato com as substâncias psicoativas, o álcool foi identificado como a primeira droga utilizada pela maioria dos indivíduos $(41,9 \%)$, seguida pela maconha (35,5\%), (Tabela 2). Silva et al. (2014), no estado de São Paulo em 2014, identificaram o álcool como sendo a substância psicoativa mais utilizada $(72,0 \%)$ e a maconha a segunda substância mais consumida $(32,0 \%)$. Em estudo semelhante realizado por Ribeiro e Carvalho (2015), avaliaram a frequência do uso de drogas em dois grupos de pacientes estudados nos CAPS-AD em Curitiba - PR, no ano de 2015, constataram que todos os participantes faziam uso de álcool $(100 \%)$, e o tabaco $(82,6 \%)$, a maconha foi à terceira substância mais utilizada $(77,7 \%)$ entre os dependentes. Resultados diferentes são encontrados na pesquisa de Santos et al. (2014), no CAPS AD II de Parnamirim-RN, nesta pesquisa a maioria utilizava o crack (64\%) como primeira escolha e o álcool (61\%) como a segunda substância mais utilizada.

Para Guimarães et al. (2004), um dos motivos que sustenta a ingestão elevada de álcool e maconha é a facilidade para adquirir, tendo em vista que são dois produtos de fácil acesso, sendo que o álcool mais ainda por ser uma droga lícita. 
Com relação à motivação para o uso de substâncias psicoativas, grande parte dos indivíduos informou ter usado drogas pela primeira vez por curiosidade $(48,4 \%)$ em seguida por influência de amigos (32,3\%), (Tabela 2). Igualmente, a pesquisa realizada por Neto et al. (2012), realizada na cidade do Porto em Portugal evidenciou que o principal motivo para experimentar drogas foi à curiosidade $(77,5 \%)$. Santos et al. (2014), observaram que o motivo principal para o uso de drogas pela primeira vez foi a influencia de amigos (39\%).

No que se refere à associação de substâncias psicoativas, a maior parte dos usuários costumavam usar duas ou mais drogas ao mesmo tempo $(77,4 \%)$, (Tabela 2). Almeida et al. (2014), verificaram que a maioria dos usuários consumiam duas ou mais drogas $(34,42 \%)$. Igualmente, foi encontrado na pesquisa realizada por Silva e Lago (2019) no CAPS AD III em uma cidade da Amazônia Ocidental brasileira, onde os autores constataram que a maioria dos usuários fazia uso de duas ou mais drogas $(85,0 \%)$.

De acordo com Macagnan et al. (2014), a ausência da droga e o desejo de ocupar o vazio deixado por uma determinada substância, fazem com que os indivíduos acabem associando diversos tipos de substâncias psicoativas.

TABELA 2 - Características relacionadas ao uso de substâncias psicoativas por usuários atendidos em um centro de referência de Rio Branco, Acre, Brasil, 2019.

\begin{tabular}{llr}
\hline Variável & N & $\%$ \\
\hline Histórico familiar & 20 & \\
Sim & 11 & 64,5 \\
Não & & 35,5 \\
Primeira substância & & \\
psicoativa utilizada & 01 & 3,2 \\
Tabaco & 13 & 41,9 \\
Álcool & 11 & 35,5 \\
Maconha & 02 & 6,5 \\
Cocaína & 02 & 6,5 \\
Crack & 02 & 6,5 \\
Outras & & \\
Motivação para o uso de & & \\
substância psicoativa & 02 & 6,5 \\
Problemas familiares & 04 & 12,9 \\
Problemas emocionais & 10 & 32,2 \\
Influência de amigos & 15 & 48,3 \\
Curiosidade & & 77,4 \\
Associação de psicoativos & 24 & 22,6 \\
Sim & 07 & $\mathbf{1 0 0 , 0}$ \\
Não & $\mathbf{3 1}$ & \\
\hline Total & & \\
\hline
\end{tabular}

Conforme a tabela 3, a maior parte dos indivíduos relatou se sentir excluído da sociedade por serem dependentes químicos $(77,4 \%)$. De acordo com Zannata et al. (2012), o sentimento de exclusão exposto pelos usuários de drogas é causado pelo preconceito da sociedade para com os dependentes químicos, circunstâncias essas que contribuem para a desvalorização social e influencia a forma como são vistos. 
Com relação à autoavaliação de saúde, a maioria $(58,1 \%)$ dos usuários avaliaram suas condições de saúde como regular e 19,4\% avaliaram como sendo ruim (Tabela 3). Na pesquisa de Dantas et al. (2017), na cidade de Maceió-AL, estes autores evidenciarem que a maioria dos usuários avaliaram sua saúde como muito ruim $(43,79 \%)$.

Segundo Dantas et al. (2017), o domínio psicológico, por sua vez, abarca a visão do indivíduo sobre sua imagem, pensamentos positivos e negativos, autoestima, aprendizado e memória, e 0 uso de drogas corroboram 0 comprometimento psicológico, trazendo uma diferente imagem da percepção de sua saúde.

TABELA 3 - Características sobre o tratamento dos usuários de substâncias psicoativas atendidos em um centro de referência de Rio Branco, Acre, Brasil, 2019.

\begin{tabular}{lcc}
\hline Variável & N & $\%$ \\
\hline Exclusão social & & \\
Sim & 24 & 77,4 \\
Não & 07 & 22,6 \\
Autoavaliação da saúde & 01 & 3,2 \\
Muito boa & 06 & 19,4 \\
Boa & 18 & 58,1 \\
Regular & 06 & 19,4 \\
Ruim & & \\
Motivação para a busca de tratamento & 05 & 16,1 \\
Intervenção familiar & 03 & 9,7 \\
Influência de amigos & 23 & 74,2 \\
Iniciativa própria & & \\
Acompanhamento familiar no & & \\
tratamento & 16 & 41,6 \\
Sim & 15 & \\
Não & & 48,4 \\
Avaliação do serviço de assistência & & 41,2 \\
psicossocial & 14 & 12,9 \\
Muito bom & 13 & $\mathbf{1 0 0 , 0}$ \\
Bom & 04 & \\
Regular & $\mathbf{3 1}$ & \\
\hline Total & &
\end{tabular}

No que diz respeito à motivação para a busca por tratamento, a maioria dos participantes informou que buscaram o tratamento por iniciativa própria $(74,2 \%)$, (Tabela 3). Do mesmo modo, no trabalho realizado por Lima et al. (2011), no município de Caucaia-CE, constataram que $59,8 \%$ dos usuários buscaram tratamento de forma espontânea. Em pesquisa realizada por Trevisan e Castro (2019), nos CAPS AD da região do Triangulo Mineiro, estes observaram que a maioria dos usuários foi encaminhada para o tratamento (44,7\%).

Para Lima et al. (2011), quando dependentes químicos demoram buscar sozinhos o tratamento por não aceitação da importância e julgar como ineficaz, outros obstáculos podem aparecer e dificultar a reabilitação como a burocracia para 
conseguir atendimento ou recaídas enquanto aguardam nas listas de espera para um determinado serviço de saúde.

Com relação ao acompanhamento familiar no tratamento, a maioria dos dependentes químicos relatou que recebe esse apoio (51,6\%), (Tabela 3). Em outro estudo esta característica também ficou evidenciada, os autores Azevedo e Silva (2013) na cidade de Valinhos-SP, registraram que a maioria tinha 0 acompanhamento familiar (84,0\%). De mesmo modo, a pesquisa realizada por Matos (2015), no município de Araranguá-SC, verificou que $90,0 \%$ dos entrevistados recebiam acompanhamento familiar.

No estudo de Azevedo e Silva (2013), a maior parte dos usuários relatou ser importante o envolvimento familiar para sua reabilitação e a maioria dos familiares acreditava que o apoio da família é uma peça fundamental para recuperação dos usuários.

No que refere à avaliação dos serviços, os usuários classificaram as ações do centro de assistência psicossocial como muito boa $(45,2 \%)$ e boa $(41,2 \%)$, (Tabela 3). O estudo realizado por Silva et al. (2018), na cidade de Belo Horizonte-MG, no ano de 2016, concluiu que $65,0 \%$ dos usuários estavam satisfeitos ou muito satisfeitos com o atendimento recebido na unidade de atenção psicossocial.

Segundo Camatta et al. (2011), há vários fatores que influenciam a satisfação dos usuários com os serviços, dentre estes destacam-se o conforto, a privacidade e a individualidade dos sujeitos envolvidos e o próprio ambiente, pois quanto mais acolhedor for o ambiente e a equipe do centro, melhor é a satisfação dos indivíduos com os serviços ofertados.

\section{CONCLUSÃO}

Para auxiliar na prevenção, tratamento e criação de novas políticas de saúde voltadas aos dependentes químicos é necessário conhecer o perfil dos usuários de substâncias psicoativas, tendo em vista, que o paciente deve ser atendido de maneira individual respeitando sua diversidade e suas particularidades.

Dessa forma, quando a equipe de saúde de um centro de atenção psicossocial dispõe do perfil dos usuários de substâncias psicoativas é possível compreender os principais fatores que contribuem para a dependência química e quais as dificuldades encontradas no caminho da reabilitação.

\section{REFERÊNCIAS}

ALMEIDA, C.S.; LUIS, M.A.V. Características sociodemográficas e padrão de uso de crack e outras drogas em um caps ad. Revista de enfermagem UFPE on line, v. 11, n. 4, p. 1716-1723, 2017. Disponível em: <https://pdfs.semanticscholar.org/0fc9/b5c5b7a64fbd15234708aed51f0cd28bd048.p d>. Doi: 10.5205/reuol.10438-93070-1-RV.1104sup201714.

ALMEIDA, R.A.; ANJOS, U.U.; VIANNA, R.P.T.; PEQUENO, G.A. Perfil dos usuários de substâncias psicoativas de João Pessoa. Saúde debate, v. 38, n. 102, p. 526538, 2014. Disponível em: <http://www.scielo.br/pdf/sdeb/v38n102/0103-1104-sdeb38-102-0526.pdf>. Doi: 10.5935/0103-1104.20140049.

ALVAREZ, S. Q.; GOMES, G. C.; XAVIER, D. M. Causas da dependência química e suas consequências para o usuário e a família. Revista de Enfermagem UFPE On Line, v.8, n.3, p.641-648, 2014. Disponível em: 
<https://periodicos.ufpe.br/revistas/revistaenfermagem/article/download/9720/9804> Doi: 10.5205/reuol.5149-42141-1-SM.0803201419.

AZEVEDO, C.S.; SILVA, R.S. A importância da família no tratamento do dependente químico. Encontro: Revista de psicologia, v. 16, n. 25, p. 151-162, 2013. Disponível

em:

<https://revista.pgsskroton.com.br/index.php/renc/article/view/2439/2337>

BASTOS, F. I. P. M. VASCONCELLOS, M. T. L.; BONI, R. B.; REIS, N. B.; COUTINHO, C. F. S. (Orgs.). III Levantamento Nacional sobre o uso de drogas pela população brasileira. Rio de Janeiro: FIOCRUZ/ICICT, 2017. 528 p. Disponível em: <https://www.arca.fiocruz.br/handle/icict/34614>.

BRASIL. SECRETARIA NACIONAL DE POLÍTICAS SOBRE DROGAS. Relatório Brasileiro Sobre Drogas. 1.ed. Brasília: SENAD, p.364, 2009. Disponível em: $<$ https://www.justica.gov.br/central-de-conteudo/politicas-sobre-drogas/relatoriospoliticas-sobre-drogas/relatoriobrasileirosobredrogas-2010.pdf/@@download/file>.

CAMATTA, M. W.; NASI, C.; ADAMOLI, A.N.; KANTORSKI, L.P.; SCHNEIDER, J.F. Avaliação de um Centro de Atenção Psicossocial: O Olhar da Família. Ciência \& Saúde Coletiva, v.16, n.11, p.4405-4414, 2011. Disponível em: <http://www.scielo.br/pdf/csc/v16n11/a13v16n11.pdf>.

CANTARELLI, N.D.C.; MARCHESAN, E.K.R.; AMARAL, M.C.; LEMOS, J.C. Perfil dos usuários de substâncias psicoativas de um Hospital Universitário do Rio Grande do Sul. Revista saúde (Santa Maria), v. 40, n.1, p. 85-90, 2014. Disponível em: <https://periodicos.ufsm.br/revistasaude/article/view/12777/pdf_1>.

CAPISTRANO, F. C.; FERREIRA, A.C.Z.; SILVA, T.L.; KALINKE, L.P.; MAFTUM, M.A. Perfil sociodemográfico e clínico de dependentes químicos em tratamento: análise de prontuários. Escola Anna Nery Revista de Enfermagem, v.17, n.2, p. 234-241, 2013. Disponível em: <http://www.scielo.br/pdf/ean/v17n2/v17n2a05.pdf>.

DANTAS, F. S.; VERAS, M.O.; CAVALCANTE, J.C.; BITTENCOURT, C.C.B.L.D.; ASSIS, T.A.L. Impacto do uso de drogas na qualidade de vida de usuários: diferença entre os sexos. Revista Brasileira de Qualidade de Vida, v. 9, n.2, p.178-192, $2017 . \quad$ Disponível

em:

$<$ https://periodicos.utfpr.edu.br/rbqv/article/download/5982/4399>.

ELICKER, E.; PALAZZO, L.S; AERTS, D.R.G.C; ALVES, G.G; CÂMARA, S. Uso de álcool, tabaco e outras drogas por adolescentes escolares de Porto Velho-RO, Brasil. Epidemiologia e Serviços de Saúde, v. 24, n. 3, p. 399-410, 2015. Disponivel em: <http://scielo.iec.gov.br/pdf/ess/v24n3/v24n3a06.pdf>. Doi: 10.5123/S1679-49742015000300006.

GUIMARAES, J. L.; GODINHO, P.H.; CRUZ, R.; KAPPANN, J.I.; JUNIOR, L.A.T. Consumo de drogas psicoativas por escolares adolescentes de Assis, SP. Revista de Saúde Pública, v.38, n.1, p.130-132, 2004. Disponível em: <http://www.scielo.br/pdf/rsp/v38n1/18462.pdf> 
INSTITUTO BRASILEIRO DE GEOGRAFIA E ESTÁTISTICA (IBGE). Rio Branco. 2019. Disponível em: <https://cidades.ibge.gov.br/brasil/ac/rio-branco/panorama>.

LIMA, H. P.; MACEDO, J.Q.; BRAGA, V.A.B.; LEMOS, A.M.; JÚNIOR, I.A.S. Caracterização de Drogaditas Atendidas em Centro de Atenção Psicossocial Álcool e outras Drogas - Estudo Documental. Online Brazilian Journal of Nursing (online), v.10, n.2, 15p., $2011 . \quad$ Disponível em: <http://objnursing.uff.br/index.php/nursing/article/download/3257/966>.

MACAGNAN, J.P.; MENETRIER, J.V.; BORTOLOTI, D.S. Perfil dos usuários de um Centro de Atenção Psicossocial no município de Francisco Beltrão - Paraná. Biosaúde, v.16, n.2, p. 34-44, 2014. Disponível em: <http://www.uel.br/revistas/uel/index.php/biosaude/article/view/24354/17933>.

MARCON, S. R.; SENE, J. O.; OLIVEIRA, J. R. T. Contexto familiar e uso de drogas entre adolescentes em tratamento. SMAD, Revista Eletrônica Saúde Mental Álcool Drogas, v.11, n.3, p.122-128, 2015. Disponível em: $<$ http://pepsic.bvsalud.org/pdf/smad/v11n3/pt_02.pdf>.

MASTROIANNI, F.C.; MACRIS, C.E.; GOMES, J.R.; CAMARGO, P.J. Perfil sociodemográfico de um CAPSad e sua funcionalidade segundo os usuários. Revista psicologia e saúde, v.8, n.2, p. 3-16, 2016. Disponível em: <http://pepsic.bvsalud.org/pdf/rpsaude/v8n2/v8n2a01.pdf>.Doi:http://dx.doi.org/10.20 435/2177-093X-2016-v8-n2(01).

MATOS, S. Participação da família no processo de tratamento do dependente químico. 2015. 26p. Trabalho de Conclusão de Curso da Pós-graduação em Educação e Direitos Humanos: Escola, violência e garantia de direitos Universidade do Sul de Santa Catarina, Santa Catarina, 2015. Disponível em <http://www.uniedu.sed.sc.gov.br/wp-content/uploads/2017/02/Artigo-Sabrina.pdf>.

MORAIS, A. E. B. Perfil dos usuários de um centro de atenção psicossocial álcool e drogas de São Luís/MA. 2018. 60p. Trabalho de Conclusão de Curso (Bacharelado em Enfermagem) - Universidade Federal do Maranhão, São Luís, 2018. Disponível em: $<$ https://monografias.ufma.br/jspui/bitstream/123456789/2578/1/ADYABOTELHO.pdf

NETO, C.; FRAGA, S.; RAMOS, E. Consumo de substâncias ilícitas por adolescentes portugueses. Revista de Saúde Pública, v.46, n.5, p.808-815, 2012. Disponível em: < http://www.scielo.br/pdf/rsp/v46n5/07.pdf>.

OLIVEIRA, J. F.; NASCIMENTO, E. R.; PAIVA, M. S. Especificidades de usuários (as) de drogas visando uma assistência baseada na heterogeneidade. Escola Anna Nery Revista de Enfermagem, v. 11, n. 4, p. 694-698, 2007. Disponível em: <http://www.scielo.br/pdf/ean/v11n4/v11n4a22.pdf>.

PECHANSKY, F.; SZOBOT, C. M.; SCIVOLETTO, S. Uso de álcool entre adolescentes: conceitos, características epidemiológicas e fatores etiopatogênicos. 
Revista Brasileira de Psiquiatria, v.26, supl. 1, p. 14-17, 2004. Disponível em: $<$ http://www.scielo.br/pdf/rbp/v26s1/a05v26s1.pdf>.

RIBEIRO, D.R.; CARVALHO, D. S. O padrão de uso de drogas por grupos em diferentes fases de tratamento nos Centros de Atenção Psicossocial Álcool e Drogas (CAPS-AD). Jornal Brasileiro de Psiquiatria, v.64, n.3, p.221-229, 2015. Disponível em: <http://www.scielo.br/pdf/jbpsiq/v64n3/0047-2085-jbpsiq-64-30221.pdf>. Doi: 10.1590/0047-2085000000082

SANTOS, R.C.A.; CARVALHO, S.R.; MIRANDA, F.A.N. Perfil socioeconômico e epidemiológico dos usuários do Centro de Atenção Psicossocial Álcool e Drogas II de Parnamirim, RN, Brasil. Revista brasileira de pesquisa e saúde, v. 16, n. 1, p. 105-111, $2014 . \quad$ Disponível em: <http://periodicos.ufes.br/RBPS/article/view/8497/5993>.

SILVA, M. L.; GUIMARÃES. C. F.; SALLES. D. B. Fatores de Risco e Proteção à Recaida na percepção de Usuarios de Substancias Psicoativas. Revista Rene, v.15, n.6, p.1007-1015, 2014. Disponível em: <http://repositorio.ufc.br/bitstream/riufc/11324/1/2014_art_mlsilva.pdf>. Doi: 10.15253/2175-6783.2014000600014

SILVA, P.K.N.; LAGO, R.R. Perfil de usuários e características relacionadas à forma de busca pelo centro de atenção psicossocial álcool e outras drogas (CAPS AD). In: SOUZA, O.F. (Org.). Saúde Publica na Amazônia Ocidental. Acre: Strictu Sensu, 2019. p.76-91 Disponível em: <https://sseditora.com.br/wp-content/uploads/SaúdePública-na-Amazônia-Ocidental.pdf>.

SILVA, S.N; LIMA, M. G.; RUAS, C. M. Avaliação de Serviços de Saúde Mental Brasileiros: satisfação dos usuários e fatores associados. Ciência e saúde coletiva, v. 23, n. 11, p. 3799-3810, $2018 . \quad$ Disponível em: <http://www.scielo.br/pdf/csc/v23n11/1413-8123-csc-23-11-3799.pdf>. Doi: 10.1590/1413-812320182311.25722016

TREVISAN, E.R.; CASTRO, S.S. Centros de Atenção Psicossocial - álcool e drogas: perfil dos usuários. Saúde debate, v. 43, n.121, p. 450-463, 2019. Disponível em: <http://www.scielo.br/pdf/sdeb/v43n121/0103-1104-sdeb-43-121-0450.pdf>. Doi: 10.1590/0103-1104201912113

UNITED NATIONS OFFICE ON DRUGS AND CRIME (UNODC). World Drug Report 2019. 2019. Disponível em: <https://www.unodc.org/wdr2019/>.

WHITEFORD, H. A.; DEGENHARDT, L.; REHM, J.; BAXTER, A. J.; FERRARI, A. J.; ERSKINE, H. E.; CHARLSON, F. J. et al. Global burden of disease attributable to mental and substance use disorders: findings from the Global Burden of Disease Study 2010. The Lancet, v. 382, p. 1575-86, 2013. Disponível em: <https://www.ncbi.nlm.nih.gov/pubmed/25658103>. Doi: 10.1016/S01406736(13)61611-6. 
ZANNATA, A.B.; GARGHETTI, F.C.; LUCCA, S.R. O Centro de atenção psicossocial álcool e drogas sob a percepção do usuário. Revista Baiana de Saúde Pública, v.36, n.1, p.225-237, 2012. Disponível em:<http://files.bvs.br/upload/S/01000233/2012/v36n1/a3011.pdf >. 